Research Paper:

\title{
Evaluating the Psychometric Properties of Emotion Reactivity Scale in Iranian Adolescents: Relation to Nonsuicidal Self-Injury
}

\author{
Maryam Izadi-Mazidi ${ }^{1^{*}}$, Hamid Yaghubi ${ }^{1}$, Pavaneh Mohammadkhani ${ }^{2}$, Hamidreza Hassanabadi ${ }^{3} \mathrm{C}$ \\ 1. Department of Clinical Psychology, Faculty of Humanities, Shahed University, Tehran, Iran. \\ 2. Department of Clinical Psychology, University of Social Welfare and Rehabilitation Sciences, Tehran, Iran. \\ 3. Department of Educational Psychology, Faculty of Psychology and Education, Kharazmi University, Tehran, Iran.
}

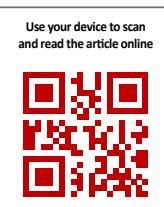

Citration Izadi-Mazidi M, Yaghubi H, Mohammadkhani P, Hassanabadi H. Evaluating the Psychometric Properties of Emotion Reactivity Scale in Iranian Adolescents: Relation to Nonsuicidal Self-Injury. Avicenna J of Neuropsychophysiology. 2017; 4(4):163169. http://dx.doi.org/10.32598/ajnpp.4.4.163

http://dx.doi.org/10.32598/ajnpp.4.4.163

수 0

Article info:

Received: 03 Apr 2017

Accepted: 21 Sep 2017

Available Online: 01 Nov 2017

Keywords:

Adolescent, Emotion, Psychometrics, Self-injurious behavior

\section{A B STRACT}

Background: Emotional reactivity is important in the development and maintenance of psychopathology, including Nonsuicidal Self-Injury (NSSI), Emotion Reactivity Scale (ERS) is a selfreport measure to assess this concept.

Objectives: The present study aimed to evaluate the psychometric properties of ERS in Iranian adolescents. We also compared the emotional reactivity in adolescents with and without NSSI.

Materials and Methods: The study samples consisted of 646 high school students with the Mean $\pm S D$ age of $16.55 \pm 0.71$ years. The data were gathered using ERS, Depression Anxiety Stress Scales (DASS), and Functional Assessment of Self-Mutilation (FASM). The collected data were analyzed using factor analysis, Pearson's correlation coefficient, and analysis of variance. Cronbach's alpha coefficient was also calculated as a measure of internal consistency.

Results: The 21-item ERS demonstrated strong internal consistency $(\alpha=0.92)$, and factor analysis supported the single factor structure of ERS. With regard to convergent validity, the ERS indicated positive correlations of medium to large magnitudes with the measures of depression, anxiety, and stress, ranging from 0.45 to 0.62 . The study participants with a history of NSSI reported significantly higher emotion reactivity, compared to those without such condition.

Conclusion: This investigation suggested that the Persian version of ERS is a reliable and valid tool for measuring emotional reactivity in nonclinical Iranian adolescents.

* Corresponding Author:

Maryam Izadi-Mazidi, PhD.

Address: Department of Clinical Psychology, Faculty of Humanities, Shahed University, Tehran, Iran.

Tel: +98 (917) 1346040

E-mail:maryam.izadi.psy@gmail.com 


\section{Introduction}

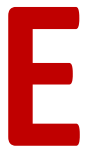

motion reactivity refers to the extent that emotion may be intensely experienced (i.e. emotion intensity), the period of time before arousal level returns to baseline (i.e. emotion persistence), and the stimulus range that trigger response (i.e. emotion sensitivity) [1]. There is a close connection between emotional reactivity and emotion regulation; more intense emotions are likely to be harder to regulate and poorer regulation skills and failure in emotional regulation are likely to result in more intense and persistent emotional responses [2-4].

A large body of literature has considered the role of emotion regulation in the development and maintenance of psychopathology [5]; however, limited studies have specifically focused on emotional reactivity. Some research has studied emotion reactivity as a component of temperament and personality [6-9]; however, temperament is a distinct and much broader component which encompasses the emotional reactivity [1]. This trend is unfortunate, as emotional reactivity may help explain why or how psychopathology is developed and maintained [1]. For instance, studies have revealed a relationship between emotional reactivity and engaging in Nonsuicidal Self-Injury (NSSI) [1, 10-13].

NSSI indicates intentional self-injuries, such as cutting, burning, biting oneself, etc. for motivations other than to die; it is socially unacceptable [14]. Adolescents are the most vulnerable group for NSSI; in a meta-analysis NSSI prevalence rate was reported equal to $17.2 \%$ among nonclinical adolescent samples [15]. Gholamrezaei et al. reported a lifetime prevalence rate of $21.3 \%$ in Iranian university students; the majority of them were engaged in NSSI from adolescence [16]. This prevalent behavior can result in serious physical consequences, such as physical injury, infectious diseases, medical complications, and sudden death [17-19]. Moreover, it leads to emotional distress $[18,19]$ and impairments in academic performance $[17,20]$, and is a potent predictor of suicidal attempts [21, 22].

Because of some problems underlying the assessment of emotional reactivity, this important construct has received little attention [23]. Nock et al. developed the Emotional Reactivity Scale (ERS) in response to the lack of specificity of previous measures (e.g. EATQ, BIS/ BAS) or the narrow focus of some of them (e.g. AIM, EIS; focusing exclusively on the intensity of emotions). ERS-a self-report instrument-was designed to assess three aspects of emotion reactivity, including intensity, persistence, and sensitivity [1].

Nock et al., and Claes et al. reported emotion reactivity was best conceptualized as a single factor construct; moreover, it demonstrated a strong internal consistency. These studies supported the construct validity of ERS $[1,13]$. The present study evaluated the psychometric properties of the Persian version of ERS. Additionally, we compared the emotional reactivity in Iranian adolescents with and without a history of NSSI.

\section{Materials and Methods}

A total of 646 students from 12 high schools in different regions of Tehran City, Iran (north, sought, west, and east), participated in the present study. Inclusion criteria included the provision of informed consent. Each subject was requested to complete FASM, ERS, and DASS12. Incomplete questionnaires were excluded from the study analysis. All the obtained personal information was kept confidential.

The sample size was calculated based on 3 to 15 subjects for each item [24] of ERS. We separately calculated the required sample size for Exploratory Factor Analysis (EFA) and Confirmatory Factor Analysis (CFA). The following instruments were applied in the current study:

\section{Emotion Reactivity Scale (ERS)}

The ERS is a self-report measure designed by Nock et al. to assess emotion reactivity. It consists of 21 items that assesses emotion sensitivity (items 3, 4, 6, 17, 19, 20,21 ), intensity (items $2,5,7,9,12,13,14,15,16,18$ ), and persistence (items $1,8,10,11$ ). Each item is rated on a 5-point Likert-Type Scale ranging from 0 (=does not describe me at all) to 4 (=describes me completely) [1]. In the study by Nock et al. the internal consistency of the 21-item ERS was reported as 0.94. Although Exploratory Factor Analysis (EFA) and reliability analysis supported a single factor structure for the ERS, the authors reported acceptable internal consistency coefficients for the three hypothesized factors of emotion, ranging from 0.81 (persistence) to 0.88 (sensitivity) [1].

\section{Depression Anxiety Stress Scale-12 (DASS-12)}

The DASS is a 12-item measure that assesses anxiety, depression, and stress. Items are rated on a 4-point Likert-type scale (0-3) [25]. Responses to the DASS-12 have demonstrated good reliability and validity in high school students [26]. 


\section{Functional Assessment of Self-Mutilation (FASM)}

This instrument was designed by Lloyd et al. to assess frequency, functions, and the other characteristics of Self-Mutilation Behavior (SMB), including the degree of physical pain, the duration of time being preoccupied before engaging in $\mathrm{SMB}$, and the abuse of alcohol or drugs during self-injury [27]. The FASM consists of two sections; the first section is a checklist of 11 self-injury behaviors (cutting the skin, burning the skin, self-biting, scratching the skin, inserting objects to the nail or skin, self-punching, picking at the wound, pulling hair, erasing the skin, and self-tattooing). We deleted self-tattooing as a NSSI behavior because many Iranian adolescents are getting fashionable tattoos. Moreover, the item 'picked at a wound' may be a clinically insignificant behavior among adolescent participants [28]; therefore, we conservatively eliminated those who only endorsed this item, from further analyses.

In the second section, 22 items inquire about the reasons that one engaged in self-injury. The items are rated on a 4-point Likert-Type Scale, ranging from 0 "never", 1 "rarely", 2 "some", to 3 "often" [27]. In the present study, we only used the first section of FASM. To investigate the internal consistency, Cronbach's alpha coefficient and split-half reliability for the total scale were calculated. To determine the convergent validity of ERS, Pearson's correlation coefficient was calculated between the ERS total score and DASS-12. EFA and CFA were then conducted to examine the factorial structure of ERS. Before conducting EFA, the sample was randomly split into two halves. The EFA and CFA were performed with the first and second halves of the samples, respectively.

Model fit was evaluated using multiple indices of fit, including Chi-Squared Test $\left(\chi^{2}\right)$, Comparative Fit Index (CFI), Root Mean Square Error of Approximation (RMSEA), Incremental Fit Index (IFI), and the Non-Normed
Fit Index (NNFI). Acceptance of models was based on the following criteria: NNFI $>0.90, \mathrm{CFI}>0.90, \mathrm{RM}-$ $\mathrm{SEA}<0.06$, and IFI $>0.90[29,30]$.

Increased emotional reactivity has been proposed as the main component in some psychological disorders, including NSSI [1]; thus, the ERS scores should differ in the presence versus absence of NSSI (i.e. criterion validity). A univariate Analysis of Variance (ANOVA) was conducted to compare individuals with a history of NSSI and those without such history in the ERS total score. Statistical analyses were performed using SPSS.

\section{Results}

The Mean $\pm S D$ age of study participants was $16.55 \pm 0.71$ (range: $15-18$ ) years. The participants' gender and academic major are presented in Table 1 . The internal consistency reliability of the 21-item ERS was obtained as 0.92. Cronbach's $\alpha$ coefficients for part 1 and part 2 of the test were 0.84 and 0.88 , respectively. The correlation between the two parts of the test was equal to 0.80 .

To explore the factor structure of ERS, an EFA (with the principal component method of extraction and Varimax rotation) was performed on the first half of the samples. The results of $\mathrm{KMO}(=0.91)$ and Bartlett's Test of Sphericity $\left(\chi^{2}=2.71 ; d f=210 ; P<0.001\right)$ revealed the factorability of the collected data (Table 2). Twenty-one items loaded significantly on a single factor. The obtained factor loadings are presented in Table 3.

The single factor model emerged from EFA, and the 3-factor model suggested by Nock et al. [1] were compared on goodness-of-fit to check whether we could replicate the one-and three-factor solution of Nock et al. [1]. The single factor and 3-factor models explained $37 \%$ and $51.94 \%$ of the total variance, respectively. A

Table 1. The participants' gender and academic major

\begin{tabular}{|c|c|c|c|c|}
\hline & & \multicolumn{3}{|c|}{ No. (\%) } \\
\hline \multicolumn{2}{|c|}{ Variable } & Total & With Self-Injury & Without Self-Injury \\
\hline \multirow{3}{*}{ Gender } & Male & $294(45.51)$ & 76(42.69) & $218(46.58)$ \\
\hline & & & & \\
\hline & Female & $352(54.48)$ & $102(57.30)$ & $250(53.41)$ \\
\hline \multirow{3}{*}{ Academic major } & Mathematics & 198(30.1) & $37(20.78)$ & $161(34.4)$ \\
\hline & Human sciences & $253(40.0)$ & $92(51.68)$ & $161(34.4)$ \\
\hline & Experimental sciences & $181(28.6)$ & $41(23.03)$ & $140(29.91)$ \\
\hline
\end{tabular}


Table 2. KMO and Bartlett's Test results

\begin{tabular}{cc}
\hline Tests & Results \\
\hline Kaiser-Meyer-Olkin Measure of sampling adequacy & 0.91 \\
Bartlett's test of Sphericity & Chi-Squared $\left(\chi^{2}\right)=2.71$ \\
$\mathrm{df}=210$ \\
$\mathrm{P}<0.001$
\end{tabular}

AJNPP

summary of the CFA results for the two models is presented in Table 4. The obtained data revealed that both models had relatively acceptable model fit; however, some criteria suggested that a single factor of emotion reactivity best represented the data, as follows: the first factor accounted for $37 \%$ of the total variance in scores, the correlation between factors was high, and all items had loadings of $\geq 0.47$ in the single factor model.

We tested the convergent validity of ERS by examining the correlation between this measure and DASS-12. The ERS suggested the positive correlation of large magni-

Table 3. Standardized factor loadings of ERS items

\begin{tabular}{|c|c|c|}
\hline Number & Item & Factor Loading \\
\hline 14 & I am easily agitated. & 0.74 \\
\hline 21 & Other people tell me I'm overreacting. & 0.68 \\
\hline 16 & When something bad occurs, my mood changes very quickly. People tell me I have a very short fuse. & 0.67 \\
\hline 13 & I am often bothered by things that other people don't react to. & 0.66 \\
\hline 12 & I get angry at people very easily. & 0.66 \\
\hline 17 & People tell me that my emotions are often too intense for the situation. & 0.66 \\
\hline 15 & My emotions go from neutral to extreme in an instant. & 0.66 \\
\hline 5 & I tend to get very emotional very easily. & 0.65 \\
\hline 20 & I often get so upset it's hard for me to think straight. & 0.63 \\
\hline 3 & When I experience emotions, I feel them very strongly/intensely. & 0.62 \\
\hline 4 & When I'm emotionally upset, my whole body gets physically upset, as well. & 0.62 \\
\hline 19 & My moods are very strong and powerful. & 0.61 \\
\hline 11 & When I am angry/upset, it takes me much longer than most people to calm down. & 0.61 \\
\hline 9 & Even the littlest things make me emotional. & 0.58 \\
\hline 1 & When something occurs that upsets me, it's all I can think about for a long time. & 0.53 \\
\hline 2 & My feelings get hurt, easily. & 0.53 \\
\hline 8 & When I feel emotional, it's hard for me to imagine feeling any other way. & 0.53 \\
\hline 6 & I experience emotions very strongly. & 0.53 \\
\hline 18 & I am a very sensitive person. & 0.53 \\
\hline 7 & I often feel extremely anxious. & 0.51 \\
\hline 10 & If I have a disagreement with someone, it takes me so long to get over it. & 0.47 \\
\hline
\end{tabular}


Table 4. Summary of the CFA results of the two models of ERS

\begin{tabular}{cccccccc}
\hline Model & $\boldsymbol{\chi}^{2}$ & CFI & NFI & NNFI & GFI & IFI & RMSEA (90\% CI*) \\
\hline 1-Factor & 911.31 & 0.95 & 0.93 & 0.94 & 0.80 & 0.95 & 0.01 \\
3-Factor & 888.83 & 0.95 & 0.93 & 0.94 & 0.80 & 0.95 & 0.01 \\
\hline
\end{tabular}

* Confidence internal

Table 5. Comparison of the emotional reactivity in individuals with and without NSSI

\begin{tabular}{|c|c|c|c|c|}
\hline \multirow{2}{*}{ Variable } & \multicolumn{2}{|c|}{ Mean士SD } & \multirow{2}{*}{$\mathbf{F}_{1,644}$} & \multirow{2}{*}{$\mathbf{P}$} \\
\hline & With NSSI & Without NSSI & & \\
\hline Emotional reactivity & $62.63 \pm 16.64$ & $49.98+15.8$ & 80.17 & $<0.001$ \\
\hline
\end{tabular}

AJNPP

tude with the total score of DASS-12 ( $r=0.62 ; \mathrm{P}<0.001)$. Correlations between the ERS and DASS-depression, DASS-anxiety, and DASS-stress were of medium to large magnitudes $(0.45,0.47$, and 0.62 respectively; $P<0.001)$. A univariate ANOVA was conducted to compare emotional reactivity between individuals with and without NSSI. As per Table 5, the study participants with a history of engaging in NSSI reported significantly higher emotion reactivity than those without such history. $\left(F_{1}\right.$ : 644=80.17, $\mathrm{P}<0.001)$.

\section{Discussion}

The present study evaluated the psychometric properties of the Persian version of ERS in Iranians high school students. With regard to the internal consistency, this measure indicated excellent internal consistency. This finding is consistent with the studies by Nock et al., Secer et al. and Claes et al. who reported strong internal consistency for ERS $[1,13,31]$.

We also observed that ERS possess a unidimensional factor structure. In two previous investigations [1, 13], also, a single factor of emotion reactivity provided the best fit to data. However, Secer et al. argued that a three-factor model best characterized the data [31]. The current study supported the convergent validity of ERS; emotional reactivity positively correlated with the measures of depression, anxiety, and stress. In line with our results, Nock et al. found significantly higher emotion reactivity in adolescents with mood or anxiety disorders, compared to those without such disorders [1].

This finding highlights the relation between emotion reactivity and emotional regulation and replicates the results of previous studies that elevations in emotion can increase the likelihood of psychopathology [32, 33].

Along with Nock et al. and Claes et al. the construct validity of ERS supported by demonstrating that scores on the ERS differ in the presence of NSSI versus absence of this condition (i.e. criterion validity). The study participants with a history of NSSI reported significantly higher emotion reactivity, compared to those without a history of NSSI $[1,13]$.

One explanation for this finding is that avoidance mediates the relationship between NSSI and emotional reactivity or closely related constructs (e.g. negative emotionality \& negative affective intensity) [10, 34, 35]. Individuals with higher levels of emotional reactivity experience more aversive cognitive and emotional states. They may use avoidance as a coping strategy to reduce emotional arousal [10]. Engaging in NSSI is a successful, albeit temporary way to reduce aversive cognitions and avoid aversive emotions [10, 36-38]. The emotion regulation function of NSSI is central in some NSSI theories [36, 39-41].

Nock et al. reported that emotional reactivity mediated the association between psychopathology and self-injurious thoughts and behaviors [1]. However, the reason for this relationship has remained unrecognized for future studies [1]. The findings should be viewed in the context of some limitations. The study samples consisted of high school students; this means the findings should be generalized to other groups with caution. Our reliance on self-report questionnaires has the limitation of biases, including social desirability and retrospective recall. Moreover, the cross-sectional design of the study restricts our ability to conclude the direction of relations 
among the study variables. These limitations should receive more attention in future investigations.

\section{Conclusion}

The attained findings suggest ERS as a psychometrically valid and reliable instrument for the measurement of emotional reactivity in adolescents. These results would facilitate research on this important construct and may also be used by clinicians.

\section{Ethical Considerations}

\section{Compliance with ethical guidelines}

The study was approved by the Ethics Committee of Shahed University (No: IR.SHAHED.REC.1397.001).

Funding

This paper was extracted from the PhD. thesis conducted By Maryam Izadi-Mazidi from Department of Clinical Psychology, Faculty of Humanities, Shahed University.

\section{Authors' contributions}

Conceptualization, author names: Maryam Izadi, Parvaneh Mohammadkhani, Hamid Yaghubi; Methodology: All authors; Project administration,: Maryam Izadi; Formal analysis: Maryam Izadi and Hammidreza Hasanabadi; Data curation: Maryam Izadi and Hammidreza Hasanabadi; Writing-original draft: Maryam Izadi; Writing-review \& editing: Parvaneh Mohammadkhani.

\section{Conflict of interest}

The authors declared no conflicts of interests.

\section{Acknowledgments}

The authors would like to thank all the participants for their valuable collaboration in this study.

\section{References}

[1] Nock MK, Wedig MM, Holmberg EB, Hooley JM. The emotion reactivity scale: development, evaluation, and relation to self-injurious thoughts and behaviors. Behavior Therapy. 2008; 39(2):107-16. [DOI:10.1016/j.beth.2007.05.005] [PMID]

[2] Gross JJ. Emotion regulation: Conceptual and empirical foundations. In Gross JJ, editor. Handbook of Emotion Regulation. New York: Guilford Press.
[3] Gross JJ, Feldman Barrett L. Emotion generation and emotion regulation: One or two depends on your point of view. Emotion Review. 2011; 3(1):8-16. [DOI:10.1177/1754073910380974] [PMID] [PMCID]

[4] Gross JJ, Sheppes G, Urry HL. Cognition and emotion lecture at the 2010 SPSP emotion preconference: Emotion generation and emotion regulation: A distinction we should make (carefully). Cognition \& Emotion. 2011; 25(5):765-81. [DOI:10.1080/02699931.2011.555 753] [PMID]

[5] Aldao A, Nolen-Hoeksema S, Schweizer S. Emotion-regulation strategies across psychopathology: A meta-analytic review. Clinical Psychology Review. 2010; 30(2):217-37. [DOI:10.1016/j. cpr.2009.11.004] [PMID]

[6] Eisenberg N, Fabes RA, Guthrie IK, Reiser M. Dispositional emotionality and regulation: Their role in predicting quality of social functioning. Journal of Personality and Social Psychology. 2000; 78(1):136-57. [DOI:10.1037/0022-3514.78.1.136] [PMID]

[7] Kagan J. Temperament and the reactions to unfamiliarity. Child Development. 1997; 68(1):139-43. [DOI:10.1111/j.1467-8624.1997. tb01931.x] [PMID]

[8] Muris P, Ollendick TH. The role of temperament in the etiology of child psychopathology. Clinical Child and Family psychology Review. 2005; 8(4):271-89. [DOI:10.1007/s10567-005-8809-y] [PMID]

[9] Rothbart MK, Ahadi SA, Evans DE. Temperament and personality: Origins and outcomes. Journal of Personality and Social Psychology. 2000; 78(1):122-35. [DOI:10.1037/0022-3514.78.1.122] [PMID]

[10] Najmi S, Wegner DM, Nock MK. Thought suppression and selfinjurious thoughts and behaviors. Behaviour Research and Therapy. 2007; 45(8):1957-65. [DOI:10.1016/j.brat.2006.09.014] [PMID] [PMCID]

[11] Zelkowitz RL. Discriminant validity of emotion regulation and emotion reactivity and relations to non-suicidal self-injury [MSc. thesis]. Nashville, Tennessee: Vanderbilt University; 2014.

[12] Glenn CR, Blumenthal TD, Klonsky ED, Hajcak G. Emotional reactivity in nonsuicidal self-injury: Divergence between self-report and startle measures. International Journal of Psychophysiology. 2011; 80(2):166-70. [DOI:10.1016/j.ijpsycho.2011.02.016] [PMID] [PMCID]

[13] Claes L, Smits D, Bijttebier P. The Dutch version of the emotion reactivity scale. European Journal of Psychological Assessment. 2014 30:73-9. [DOI:10.1037/t28296-000]

[14] American Psychiatric Association. Diagnostic and Statistical Manual of mental disorders (DSM-5 $\left.{ }^{\circledR}\right)$. Washington: American Psychiatric Association; 2000. [DOI:10.1176/appi.books.9780890425596]

[15] Swannell SV, Martin GE, Page A, Hasking P, St John NJ. Prevalence of nonsuicidal self-injury in nonclinical samples: Systematic review, meta-analysis and meta-regression. Suicide and Life-Threatening Behavior. 2014; 44(3):273-303. [DOI:10.1111/sltb.12070] [PMID]

[16] Gholamrezaei M, De Stefano J, Heath NL. Nonsuicidal self-injury across cultures and ethnic and racial minorities: A review. International Journal of Psychology. 2017; 52(4):316-26. [DOI:10.1002/ ijop.12230] [PMID]

[17] Briere J, Gil E. Self-mutilation in clinical and general population samples: Prevalence, correlates, and functions. American Journal of Orthopsychiatry. 1998; 68(4):609-20. [DOI:10.1037/h0080369] [PMID] 
[18] Turner BJ, Austin SB, Chapman AL. Treating nonsuicidal self-injury: A systematic review of psychological and pharmacological interventions. The Canadian Journal of Psychiatry. 2014; 59(11):576-85. [DO I:10.1177/070674371405901103] [PMID] [PMCID]

[19] Nock MK, Joiner Jr TE, Gordon KH, Lloyd-Richardson E, Prinstein MJ. Non-suicidal self-injury among adolescents: Diagnostic correlates and relation to suicide attempts. Psychiatry Research. 2006; 144(1):65-72. [DOI:10.1016/j.psychres.2006.05.010] [PMID]

[20] Klonsky ED. The functions of self-injury in young adults who cut themselves: Clarifying the evidence for affect-regulation. Psychiatry Research. 2009; 166(2-3):260-8. [DOI:10.1016/j.psychres.2008.02.008] [PMID] [PMCID]

[21] Guan K, Fox KR, Prinstein MJ. Nonsuicidal self-injury as a timeinvariant predictor of adolescent suicide ideation and attempts in a diverse community sample. Journal of Consulting and Clinical Psychology. 2012; 80(5):842-9. [DOI:10.1037/a0029429] [PMID] [PMCID]

[22] Klonsky ED, May AM, Glenn CR. The relationship between nonsuicidal self-injury and attempted suicide: Converging evidence from four samples. Journal of Abnormal Psychology. 2013; 122(1):231-7. [DOI:10.1037/a0030278] [PMID]

[23] Becerra R, Campitelli G. Emotional reactivity: Critical analysis and proposal of a new scale. International Journal of Applied Psychology. 2013; 3(6):161-8. [DOI: 10.5923/j.ijap.20130306.03]

[24] Munro BH. Statistical methods for health care research. Philadelphia: Lippincott Williams \& Wilkins; 2005

[25] Lovibond SH, Lovibond PF. Manual for the depression anxiety stress scales. Sydney: Psychology Foundation of Australia; 1996. [DOI:10.1037/t39835-000]

[26] Heidari T. [Investigating the psychometric properties of the depression, anxiety and stress scale-12 (Persian)] [MA dissertation] Tehran: Shahed University; 2016.

[27] Lloyd EE, Kelley ML, Hope T. Self-mutilation in a community sample of adolescents: Descriptive characteristics and provisional prevalence rates. Paper presented at: Annual meeting of the Society for Behavioral Medicine. 19 April 1997. New Orleans, Louisiana.

[28] Lloyd-Richardson EE, Perrine N, Dierker L, Kelley ML. Characteristics and functions of non-suicidal self-injury in a community sample of adolescents. Psychological Medicine. 2007; 37(8):1183-92. [DOI:10.1017/S003329170700027X] [PMID] [PMCID]

[29] Hu LT, Bentler PM. Cutoff criteria for fit indexes in covariance structure analysis: Conventional criteria versus new alternatives. Structural Equation Modeling: A Multidisciplinary Journal. 1999; 6(1):1-55. [DOI:10.1080/10705519909540118]

[30] Bentler PM, Bonett DG. Significance tests and goodness of fit in the analysis of covariance structures. Psychological Bulletin. 1980; 88(3):588-606. [DOI:10.1037/0033-2909.88.3.588]

[31] Seçer I, Halmatov S, Gençdoğan B. [Adaptation of emotional reactivity scale to Turkish: Reliability and validity study (Turkish)]. Sakarya University Journal of Education. 2013; 3(1):77-89.

[32] Davidson RJ, Putnam KM, Larson CL. Dysfunction in the neura circuitry of emotion regulation- A possible prelude to violence. Science. 2000; 289(5479):591-4. [DOI:10.1126/science.289.5479.591] [PMID]

[33] Lynch TR, Cheavens JS, Morse JQ, Rosenthal MZ. A model predicting suicidal ideation and hopelessness in depressed older adults:
The impact of emotion inhibition and affect intensity. Aging \& Mental Health. 2004; 8(6):486-97. [DOI:10.1080/136078604123313037 75] [PMID]

[34] Rosenthal MZ, Cheavens JS, Lejuez CW, Lynch TR. Thought suppression mediates the relationship between negative affect and borderline personality disorder symptoms. Behaviour Research and Therapy. 2005; 43(9):1173-85. [DOI:10.1016/j.brat.2004.08.006] [PMID]

[35] Kingston J, Clarke S, Remington B. Experiential avoidance and problem behavior: A mediational analysis. Behavior Modification. 2010; 34(2):145-63. [DOI:10.1177/0145445510362575] [PMID]

[36] Chapman AL, Gratz KL, Brown MZ. Solving the puzzle of deliberate self-harm: The experiential avoidance model. Behaviour Research and Therapy. 2006; 44(3):371-94. [DOI:10.1016/j.brat.2005.03.005] [PMID]

[37] Bentley KH, Sauer-Zavala S, Wilner J. The unique contributions of distinct experiential avoidance domains to severity and functionality of non-suicidal self-injury. Journal of Experimental Psychopathology. 2015; 6(1):40-57. [DOI:10.5127/jep.040613]

[38] Howe-Martin LS, Murrell AR, Guarnaccia CA. Repetitive nonsuicidal self-injury as experiential avoidance among a community sample of adolescents. Journal of Clinical Psychology. 2012; 68(7):80929. [DOI:10.1002/jclp.21868] [PMID]

[39] Klonsky ED. The functions of deliberate self-injury: A review of the evidence. Clinical Psychology Review. 2007; 27(2):226-39. [DOI:10.1016/j.cpr.2006.08.002] [PMID]

[40] Nock MK, Prinstein MJ. A functional approach to the assessment of self-mutilative behavior. Journal of Consulting and Clinical Psychology. 2004; 72(5):885-90. [DOI:10.1037/0022-006X.72.5.885] [PMID]

[41] Nock MK. Why do people hurt themselves? New insights into the nature and functions of self-injury. Current Directions in Psychological Science. 2009; 18(2):78-83. [DOI:10.1111/j.14678721.2009.01613.x] [PMID] [PMCID] 